\title{
CT Dynamics: The Shift from Morphology to Function
}

\author{
Rich Mather
}

Published online: 12 January 2013

(c) Springer Science+Business Media New York 2013

\begin{abstract}
CT has historically been a static imaging modality, but the human body is in constant motion. The need to visualize the underlying physiology has driven CT to capture functional information as well. CT dynamics can be acquired using several different acquisition techniques on both conventional and high-end scanners. Dynamic joints, dynamic CTA, perfusion, and dynamic lungs are all emerging applications of CT dynamics. The use of dynamic CT can yield key diagnostic information not available from static scans.
\end{abstract}

Keywords Computed tomography .

Dynamic volume CT . Dual source CT .

320-row CT · Dynamic imaging

\section{Introduction}

Since its beginnings as solely a neurological imaging device, the number of applications of CT imaging has expanded tremendously. Currently, CT has applications for every anatomical region and nearly every physiological process. CT technology has seen both gradual improvements and breakthrough advances driven by accuracy and sensitivity as well as in speed and economic efficiency. The use of CT has steadily increased by more than threefold since 1993 to over 70 million scans per year [1]. By and large, however, CT has traditionally been used as a

R. Mather $(\bowtie)$

Toshiba Medical Research Institute, 706 N Deerpath Dr,

Vernon Hills, IL, USA

e-mail: rmather@tmriusa.com modality that produces static images of morphology with high spatial resolution and good soft tissue contrast.

Static anatomic information has always been used to infer the underlying physiology and function. One good example of this is in coronary angiography where the physiologic significance of a lesion is determined wholly by its degree of stenosis: a morphologic metric. However, landmark clinical trials such as COURAGE [2] and the FAME I [3] and FAME II [4••] trials have shown that such metrics are inadequate. COURAGE showed that the outcome of patients receiving percutaneous coronary intervention (PCI) driven solely by anatomical measures was no better than for patients who received no surgical intervention but were treated with optimal medical therapy (OMT). Subsequently, the FAME trials showed that using fractional flow reserve (FFR), a functional metric, to drive PCI led to a significant reduction in hospitalization and urgent revascularization compared to the OMT group. In order to realize this kind of higher precision imaging, earlier diagnosis, and direct impacts on patient therapy, there is currently a shift in the nature of CT applications from morphology to function. This shift in CT clinical applications towards the imaging of function has opened up a new paradigm in clinical imaging: CT dynamics.

The human body is an inherently dynamic machine. Whether examining the flow of blood through the vessels and perfusing into tissue, the expansion and contraction of the lungs, the movement of the vocal cords, or the flexion and extension of the wrist, the body is in constant motion. The clinical ability to capture that motion and monitor the anatomic and physiologic changes over time can add significant information to the diagnostic process. Dynamic joint instabilities, myocardial wall motion abnormalities, and blood flow measurements all require the imaging of volumetric anatomy over time. 


\section{Acquisition}

The idea of dynamically acquired CT data is not new. In fact, two unconventional CT designs, the Dynamic Spatial Reconstructor [5] (DSR) and the Imatron electron beam scanner [6,7] were both built in the 1980s primarily as dynamic scanners focused on cardiac imaging (Fig. 1). The DSR consisted of $28 \mathrm{x}$-ray tubes mounted in a semicircle with opposing image intensifiers and video cameras. The system could image with $z$-axis coverage of $24 \mathrm{~cm}$ with a $38 \mathrm{~cm} x-y$ field of view. The machine rotated continuously and could trade off temporal and spatial resolution. With 28 views, it could produce 60 frames per second or use all 240 views that could be acquired in a $2 \mathrm{~s}$ acquisition. While this machine had limitations with regards to cost and practicality for widespread clinical deployment, it was a pioneer in dynamic imaging. Similarly unique in design, Imatron's electron beam scanner departed from the conventional design of an x-ray tube and substituted in its place an electron beam being swept across a large semicircular tungsten target array. The electron-beam sweep provided the opportunity to produce a partial-scan temporal resolution as fast as $50 \mathrm{~ms}$. Furthermore, the Imatron system was the first volume CT system covering $8 \mathrm{~cm}$ of anatomy with no table motion. There were two detector-rows and four sets of tungsten targets for the beam to sweep over. In all, this allowed eight distinct $8 \mathrm{~mm}$ slices in the z-direction through the anatomy. By cycling through the four sets of targets repeatedly, the system was able to capture a volume of anatomy dynamically leading to the scanner's nickname: cine CT. While these early designs for dynamic CT did not ultimately persist, they did pioneer CT dynamic imaging which is currently experiencing a clinical resurgence.

Modern, commercially-available CT scanners offer several methods for acquiring dynamic CT data: low pitch helical, helical shuttle, and dynamic volume imaging. Each acquisition method provides certain advantages and limitations in the collection of data.

\section{Low-Pitch Helical}

One of the simplest ways to acquire dynamic data with a conventional helical scanner, the low-pitch helical approach can be employed for any anatomy where the motion is periodic and the anatomy returns to the same spatial position each cycle. By moving the table slowly enough to capture the same anatomy throughout its cyclical motion, it is possible to re-sort the projections from multiple different detector rows to reconstruct a given section of anatomy at any arbitrary time point in its cycle. Early work with this approach focused solely on imaging the coronary arteries [8-11], while more recent studies have used this approach with respiratory gating in the lungs [12].

The main advantage of low-pitch helical scanning is that it can be used on any multidetector row helical scanner. The technical requirements fall on the reconstruction algorithm, however, and there is a significant tradeoff in radiation dose since the technique relies on selecting the relevant projections from an overabundance of data. Also, when the assumption of periodicity is broken, the reconstructed images can contain blurring and artifacts.

\section{Helical Shuttle}

With the helical shuttle technique, the same section of anatomy is scanned periodically with a helical acquisition, typically back and forth through the anatomy [13]. With this type of acquisition, large sections of anatomy can be captured over time. The trade-off, however, is that the volumetric temporal resolution is poor, degrading as the scan distance increases. Therefore, it is not possible to capture the rapid motion of the heart, lungs, or joints with this technique. Recently however, some groups have used this technique to capture multiple phases of vascular contrast flow [14]. It is worth noting that with this technique the absolute phase of motion varies along the length of the acquisition. That is, the difference between the temporal phase at the start of the imaging volume and the end increases with increasing scan distance.

\section{Dynamic Volume}

The most direct way to measure dynamic motion, especially of rapidly moving anatomy, is by repeated scanning without table motion. This can be accomplished with any modern CT scanner, although the technique is limited by the craniocaudal or $z$-axis coverage of the system. For example, most 64-row systems can cover approximately $4 \mathrm{~cm}$ of anatomy dynamically. Other systems have greater anatomic coverage with 8 and even $16 \mathrm{~cm}$ of anatomy being acquired in a single rotation without table motion $[15,16]$. The volume scan mode allows the imaged volumes to be interrogated dynamically with temporal uniformity, i.e., the entire volume is imaged at the same temporal phase [17]. The trade-offs with the wider volume systems are an increase in scatter at the detector and the necessity of image reconstruction to handle the cone beam acquisition geometry.

\section{Clinical Applications of Dynamic CT}

With these acquisition modes, CT has now become a robust modality for the visualization of the human body in 

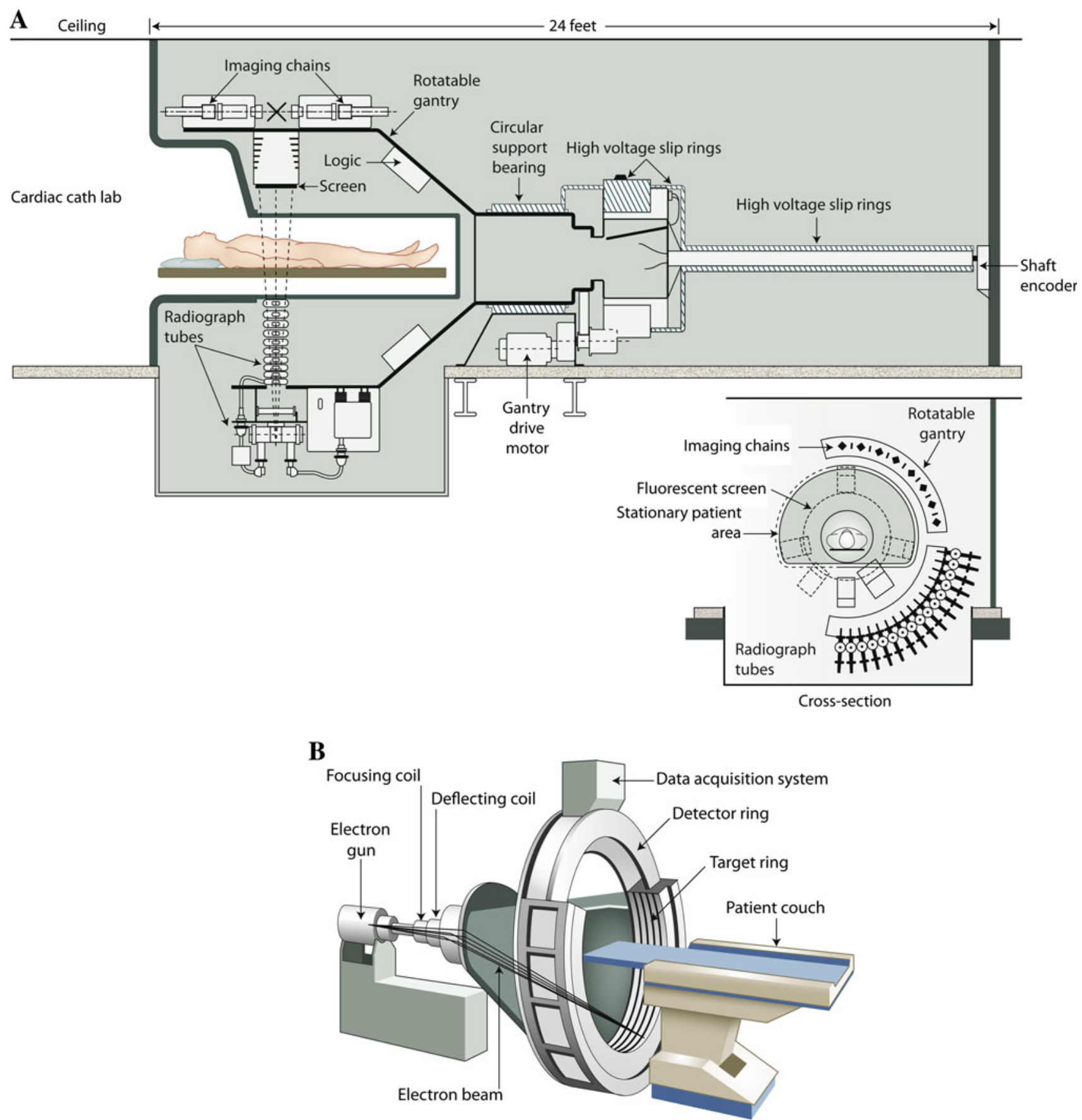

Fig. 1 Early pioneers of dynamic imaging with CT. a The Dynamic Spatial Reconstructor (DSR) was built from 28 x-ray tubes and image intensifiers. b Imatron's electron beam scanner swept an electron

motion. A number of new clinical applications have arisen with the increasing use of dynamic CT: musculoskeletal kinematics (MSK), dynamic CTA, CT perfusion, and other exams with anatomic motion. With each application, the dynamic nature of CT plays an important role. Some applications, like MSK, require high volumetric temporal sampling, while others, like perfusion, may be adaptable to lower sampling rates. beam across a large, semicircular tungsten target surrounding the patient. Figures reprinted with permission

\section{MSK Kinematics}

Joint imaging in radiology has historically focused on morphology rather than function by acquiring static images of a stationary joint. When there is an obvious deformity in the joint, this approach can be useful. However, this is often not the case, as with joint instabilities [18], where the diagnosis relies on interrogation of the joint in motion 
(Fig. 2). While there are some other non-invasive dynamic techniques available such as fluoroscopy [19] and ultrasound [20], only CT provides 4D imaging with high temporal and spatial resolution. It is possible to image a series of static positions with helical CT; however, a fluoroscopic study of the carpal bones has shown that there can be sudden changes in position which would be missed using a "stop-motion" technique [21].

While the earliest work in CT imaging of joint mechanics was conducted by Stanford et al. and Shapeero et al. [22, 23] on the electron beam scanner, early MDCT feasibility work by Tay et al. [24, 25] employed a cadaveric wrist attached to a periodic motion device and scanned with a low helical pitch and retrospective gating. While this work showed the potential of the imaging of joint motion with $\mathrm{CT}$, later work by members of the same group concluded that the imperfect periodicity inherent in patient imaging produces banding artifacts with the retrospective technique [26]. In this subsequent work, this team examined dynamic imaging of the cadaveric wrist without table motion. They showed that the dynamic imaging did not rely on periodic motion and eliminated the banding artifacts. While the longitudinal coverage for this study was only $38.4 \mathrm{~mm}$, Leng et al. found this to be sufficient for evaluation of scapholunate instability where the main focus is on the proximal carpal bones.

Kalia et al. [27•] first reported on the technical feasibility of dynamic joint imaging with a wide detector CT scanner. Using a 256 detector-row prototype system with $12.8 \mathrm{~cm}$ of longitudinal coverage, they dynamically evaluated both the knee and the wrist in vivo. Three volunteers underwent bilateral knee imaging for patellofemoral evaluation and three others underwent bilateral wrist imaging for both supination-pronation and radioulnar deviation. The investigators found that the image quality was rated as "good" in all cases with some artifacts noted. Furthermore, the ability to depict normal joint motion was rated with the highest rating of "well depicted" for all joints examined. They concluded that the wide-detector dynamic evaluation of dynamic joints was feasible and showed good potential for evaluation of pathomechanics including patellofemoral maltracking, carpal instability, and chronic distal radioulnar joint instability. This work was followed up using a 320 detector-row system with $16 \mathrm{~cm}$ of longitudinal coverage by Halpenny et al. [28] looking specifically at scapholunate instability in a patient. They demonstrated the ability to measure the increase in the scapholunate distance during the motion that caused the patient the most pain.

Recently, Wassilew et al. [29] looked at the dynamic assessment of femoroacetabular impingement and subluxation in 30 patients using the 320 detector-row scanner. They compared the results with interoperative findings and found that the dynamic CT had a high degree of accuracy and was a new option for visualizing morphology and impingement prior to surgery. The main limitation of this approach was noted to be its increased radiation dose over static, diagnostic CT of the same region.

\section{Dynamic CTA}

CTA has been shown to be a useful technique in the detection and evaluation of morphological pathologies such as vessel stenoses, aneurysms, congenital abnormalities, and arteriovenous malformations (AVM). However, with static images, it is impossible to demonstrate the dynamic properties of these pathologies such as early venous filling and the feeding and draining patterns of an AVM [30]. Further, while static CTA has been shown to be a sensitive and specific test in the detection of endoleaks [31], invasive digital subtraction angiography (DSA) remains the gold standard in their classification as well as in the characterization of flow patterns in aneurysms and AVMs. While there are several modalities that can measure flow in the vessels such as DSA, ultrasound, and dynamic MR, dynamic CTA (or 4D-CTA) investigation of these pathologies (Fig. 3) offers a non-invasive alternative to DSA, is more widely available than MR, and can access more vessels than ultrasound.

Bent et al. [32•] were the first to report on volumetric dynamic CTA evaluating its utility in the characterization of endoleaks following fenestrated endovascular aortic aneurysm repair (f-EVAR). The report argues that while DSA is the gold standard in these cases, it is a challenging procedure due to the high number of arterial puncture sites and contrast injections, as well as carrying a high radiation dose. The group used a 320-detector row system, scanning dynamically and continuously for $18 \mathrm{~s}$ with a $0.5 \mathrm{~s}$ rotation time. They were able to clearly demonstrate a type III endoleak in their patient and argued that such a demonstration would not be possible with a static CTA exam.

Brouwer et al. [33] described dynamic CTA evaluation of shunting lesions such as AVMs and DAVFs using the 320-detector row scanner. They generated 22 time points from the CT data for each case, and used the initial time point as the subtraction mask. The resulting data were compared to standard catheter angiography (CA) in the same patients. They showed comparable diagnostic ability between CA and dynamic CTA for these cases demonstrating early venous filling, cortical venous reflux, and a benign DAVF. Simultaneous visualization of all cranial vascular territories was noted using CT compared to the selective vessel injections possible with CA. An advantage of CT is that it allows the visualization of the anatomy and function from any angle in post-processing where CA views must be determined during the procedure. The maximum 

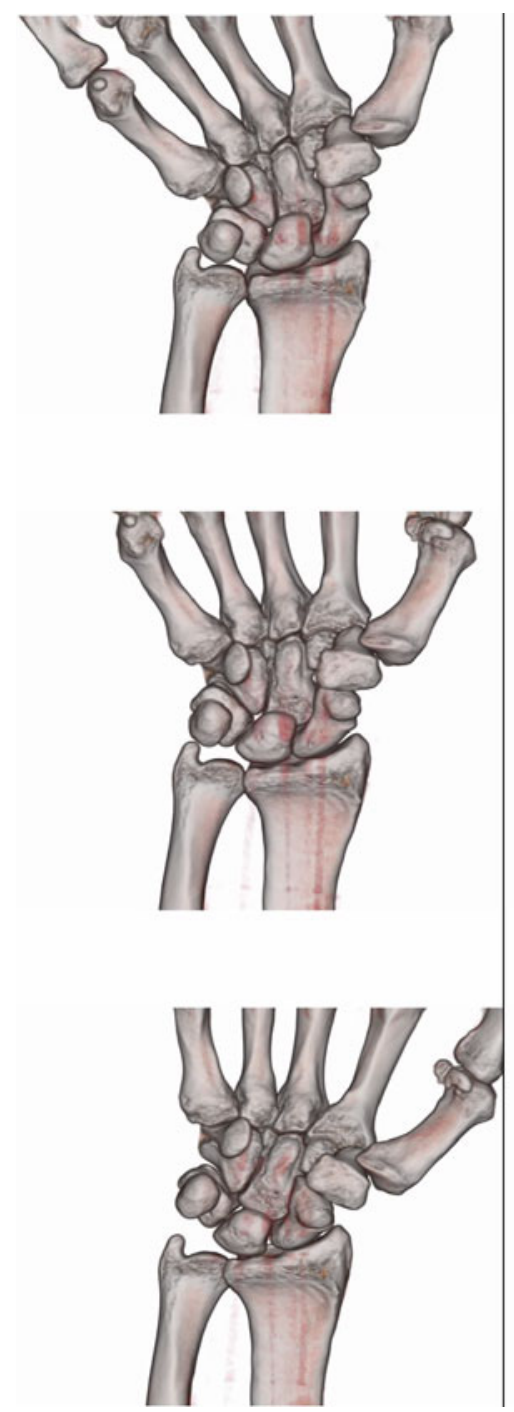
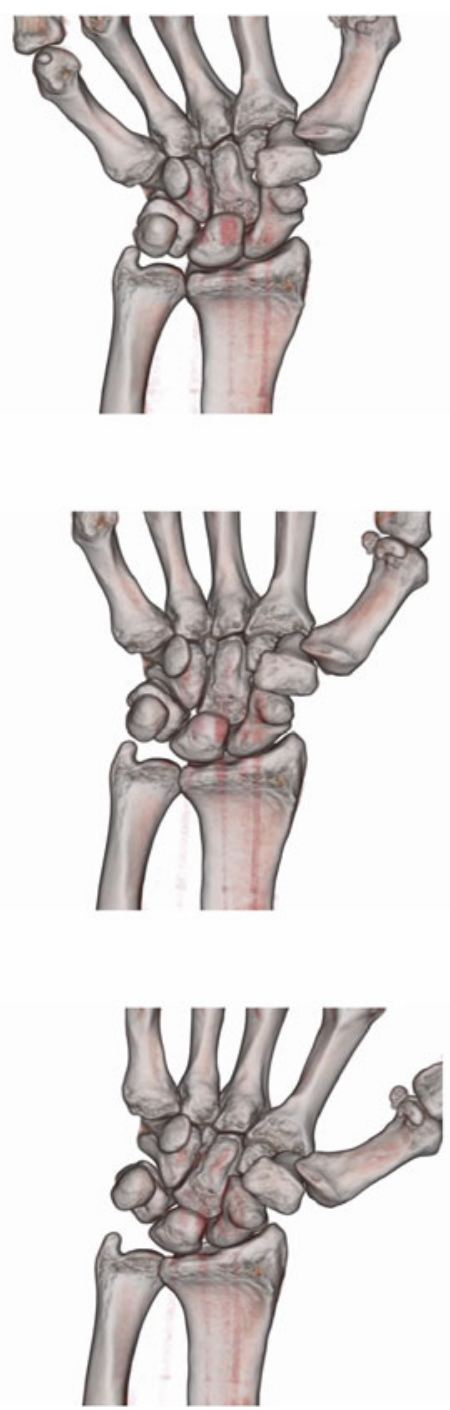
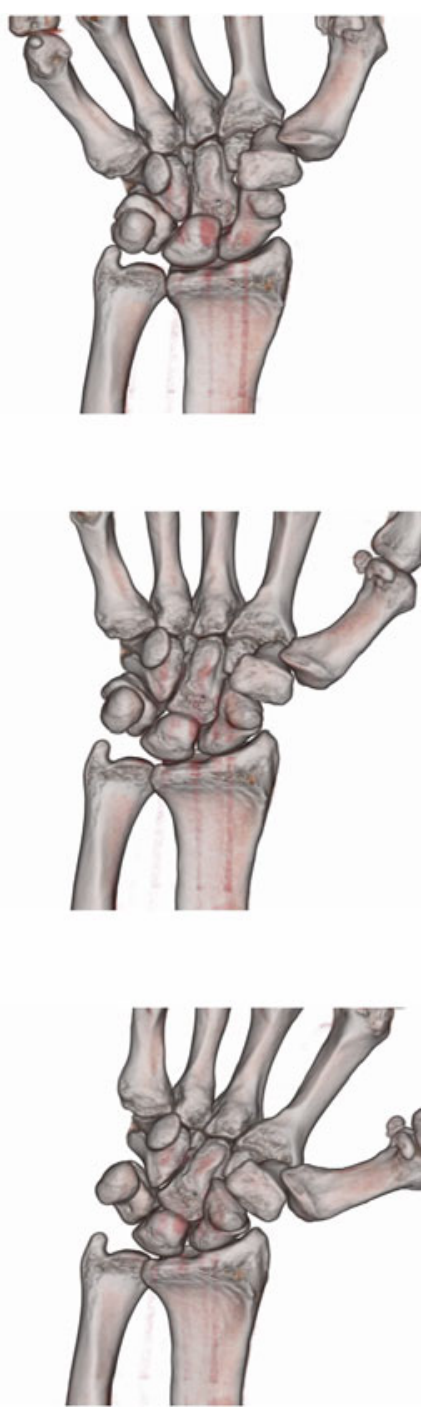

Fig. 2 Dynamically acquired musculoskeletal imaging of the wrist showing increased scapholunate distance. Data were acquired in dynamic volume mode with $16 \mathrm{~cm}$ of coverage. Images courtesy of Dr Alain Blum

radiation dose for CT was reported to be $5.1 \mathrm{mSv}$. In a follow-up study, Willems et al. [34] demonstrated high correlation between dynamic CTA and CA in the detection of arteriovenous malformations of the brain in a small cohort of 17 patients. They found the main limitations of 4D-CTA were in the accurate estimation and identification of some of the angioarchitectural details such as underestimation of the nidus size and misinterpretation of indirect feeding through pial collaterals. Ultimately, they found 4DCTA to be sufficient to accurately diagnose and classify the shunt. In a separate study, Willems et al. [35] also describe the ability of 4D-CTA to identify and classify DAVFs in a cohort of 11 patients. They found the technique to be robust unless the patient had a small, slow-flow DAVF in which case the pathology might be missed. Finally, Krings et al. [36] reported on the flow dynamics of an intracavernous aneurysm and evaluate the aneurysm's pulsatility using dynamic CT. They postulated that the focal pulsations were associated with the subsequent growth of the aneurysm in the region that showed pulsatility.

Recently, Meinel et al. [37] reported on time-resolved angiography using a dual-source scanner in a multi-phase helical shuttle exam using a technique previously described by Sommer [38•]. In this study, they scanned 14 patients with known or suspected aortic dissection using six reconstructed time phases covering $48 \mathrm{~cm}$. The time between each phase was $6 \mathrm{~s}$. They demonstrated the difference in enhancement time between the true and false lumen and the oscillation of the dissection membrane, difficult to visualize without dynamics. The average radiation dose was $27.7 \mathrm{mSv}$. Sommer et al. [39] used a similar scanning technique to detect and classify endoleaks compared to contrast enhanced ultrasound. In a cohort of 54 patients, they acquired 12 helical phases of $27 \mathrm{~cm}$ with $2.5 \mathrm{~s}$ between 

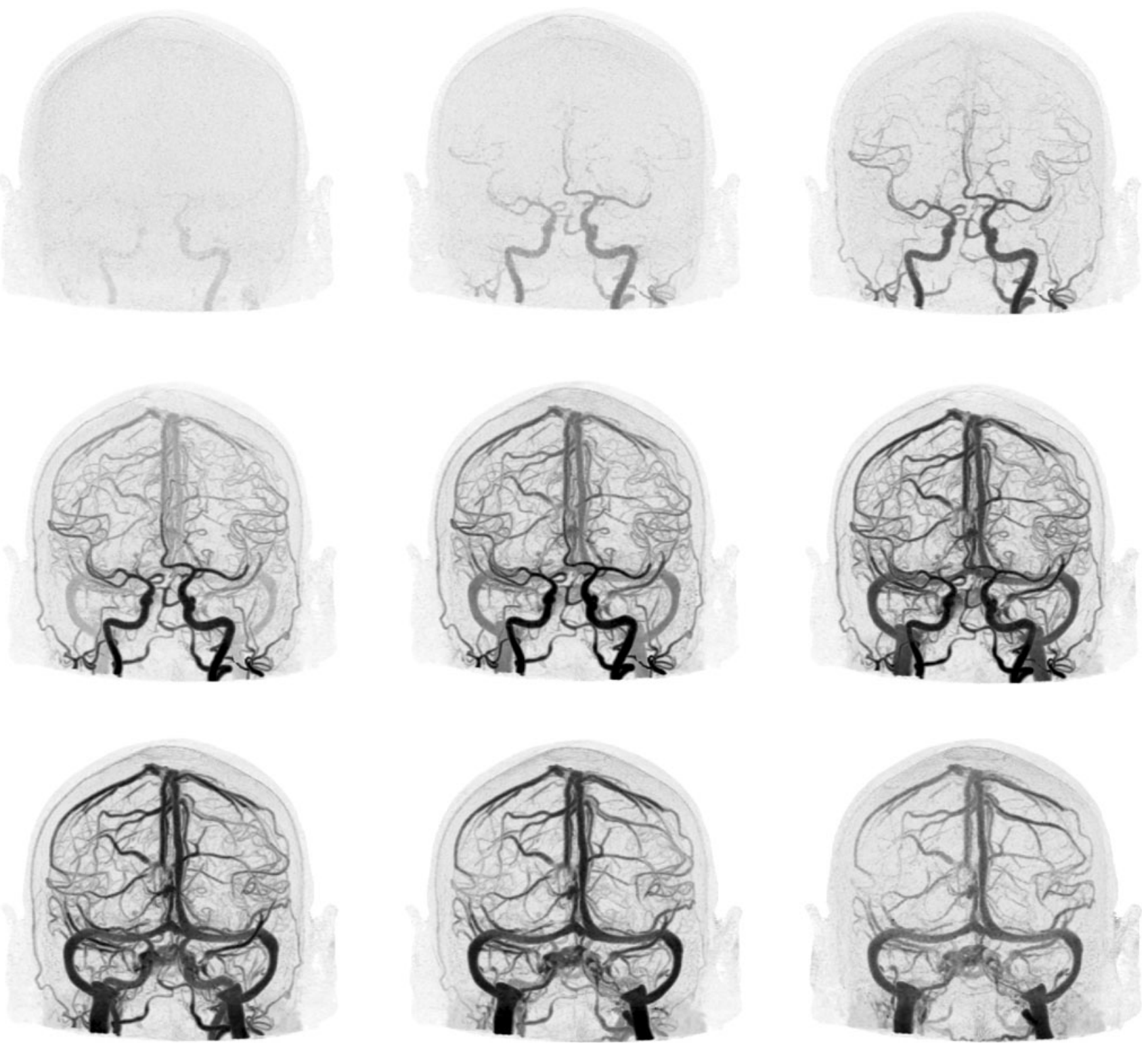

Fig. 3 Dynamic subtraction CT angiography of the whole brain. Dataset shows timing of dynamic vessel filling in 4D. Data were acquired in dynamic volume mode with $16 \mathrm{~cm}$ of coverage. Images courtesy of Toshiba Medical Systems Corporation

each phase. They showed high sensitivity and specificity for detecting and classifying primarily type I and type II endoleaks with an average radiation dose of $14.6 \mathrm{mSv}$. In a similar study, Lehmkuhl et al. examined the rate of type I and type II endoleak detection with no reference standard using, rather, a metric of diagnostic confidence. They found that endoleaks were more likely to be found in the dynamic scan which contained phases that were not typically acquired in a standard biphasic CTA exam.

Barfett et al. [40] used dynamic volume CT in a phantom to show the feasibility of measuring blood flow directly. Using a method from conventional angiography [41], the group compared the bolus profiles at two locations within a cadaveric vessel. They noted that the dynamic CT technique holds advantages over both Doppler ultrasound and phase contrast MRI in that it is rapid, user independent, and can potentially measure the flow in small vessels that are inaccessible to ultrasound. However, this technique is limited by the need for a reasonable distance between the bolus measurements to correspond to the precision of the temporal measurements, as well as by the sensitivity to different phases of the cardiac cycle.

\section{Perfusion}

Distinct from the imaging of morphological motion and vascular dynamics, perfusion imaging attempts to quantify 
the capillary flow within the parenchyma of various organs in the body. First described by Axel [42], perfusion imaging is typically accomplished in CT by analyzing the enhancement of the tissue over time as iodinated contrast washes in and out of the tissue. The plot of this enhancement over time is known as a time-density curve (TDC), and by repeated imaging of the anatomy of interest, the TDC can be sampled and flow quantified. Early CT perfusion work was pioneered on the electron beam scanner imaging the myocardium [43, 44]. More recently, CT neuroperfusion has become an invaluable application for the triage of patients with suspected stroke [45-48] (Fig. 4). CT is fast, inexpensive, and widely available, making it a viable option for stroke workup. The typical exam consists of an unenhanced acquisition to rule out hemorrhage, a CTA exam from the aortic arch to the vertex of the skull to map the head and neck vasculature, and a dynamic CT perfusion (CTP) exam to determine the level of ischemia and infarction in the brain [49]. In order to cover the entire anterior circulation territory or, better yet, the entire brain, $8-16 \mathrm{~cm}$ of $z$-axis coverage is required. This can either be accomplished with a wide volume detector scanner [50-52] or by using jog [53] or helical shuttle [54] modes of acquisition.

Currently, CTP is being used in nearly every organ of the body (Fig. 5). Liver perfusion with CT can help assess chronic liver diseases such as chronic hepatitis, liver fibrosis, and cirrhosis as well as detect primary hepatic carcinomas [55, 56]. Other recent studies have looked at reproducibility [57], assessing and lowering the radiation dose $[58,59]$, comparing to arterial spin labeling with MR [60], and have examined different analysis methods [61]. Miles et al. [62] first described the use of perfusion CT in the pancreas in 1995, but widespread application of the technique has been limited by the inability of conventional CT to cover the entire organ and the respiratory motion that can shift the organ out of the field of view. More recently, Kandel et al. [63] described the use of dynamic volume CT in the pancreas (Fig. 6) to overcome these limitations. Similarly, others have described dynamic perfusion of the pancreas using $4 \mathrm{~cm}$ of coverage [64-66]. Furthermore, there are numerous reports of the utility of perfusion [67] in the lung [68•, 69-71], kidney [72-74], spleen [75, 76], and colon [77-79]. Finally, there is a great deal of work ongoing for both dynamic [80, 81] and single-shot [82-84] myocardial perfusion.

\section{Dynamic Airways and Lungs}

While static CT has a large number of applications in the lungs and airways, the dynamic motion of this anatomy has historically been inferred from one or more scans [85].
While earlier studies by Lee et al. [86] looked at tracheomalacia during forced expiration with a static helical scan , Wagnetz et al. [87] did a similar study with dynamically acquired volume CT in six patients with a suspicion of tracheomalacia. They imaged for either 4.5 or $6.5 \mathrm{~s}$ from the end of inspiration through the forceful expiration phase. By scanning dynamically, they found that the peak airway collapse did not occur synchronously; rather, the distal trachea had peak collapse at 2-3 s into expiration where the proximal trachea showed peak collapse at 3-4 s. They reported a maximum radiation dose of $8.2 \mathrm{mSv}$ for this study. In another airway study, Low et al. [88] examined abnormal laryngeal function in patients with difficult to treat asthma. They imaged the larynx dynamically during normal breathing over the course of 1 respiratory cycle. They found abnormal narrowing in the vocal cords in $50 \%$ of the asthmatic patients compared to a cohort of healthy volunteers. The maximum dose for the dynamic study was reported to be $2 \mathrm{mSv}$.

Several reports have described the dynamic visualization (Fig. 7) of respiration in the lungs [89-91]. Some approaches use a step-and-shoot acquisition covering small volumes at a time and using an external marker to achieve respiratory gating [92]. Others use a low helical pitch, typically around 0.1 , to retrospectively gate the lungs during a helical scan. Greenberg [93] describes the use of wide detector scanning to image the pediatric lung. He found that the dynamic acquisitions often added diagnostic information that was unavailable from static scans, including a better appreciation for tracheo- and bronchomalacia and a more accurate assessment of air trapping. He reported a mean radiation dose of $1.7 \mathrm{mSv}$ in the cohort of 24 infants and small children.

\section{Conclusion}

Nearly any anatomy in motion can be imaged with dynamic CT. There is increased diagnostic information available over and above static imaging by repeatedly interrogating the anatomy during motion. Through the use of dynamic axial, low-pitch helical, shuttle mode, or wide volume scanning, anatomic dynamics can be captured and visualized with most modern CT systems. There are, however, a few caveats and limitations to the employment of these dynamic acquisitions.

One challenge is the radiation dose associated with repeated scanning of the same anatomy. Since dose is cumulative, every imaging phase multiplies the amount of energy absorbed by the patient. This is typically mitigated by using scan techniques that result in static images that are noisy and of lower quality than the standard diagnostic technique. With contrast enhanced studies, the use of a 
Fig. 4 Dynamically acquired brain perfusion exam showing core infarct and penumbral region in the left hemisphere. Individual maps show a blood flow, b blood volume, c mean transit time, and $\mathbf{d}$ tissue characterization. Images courtesy of GE Healthcare
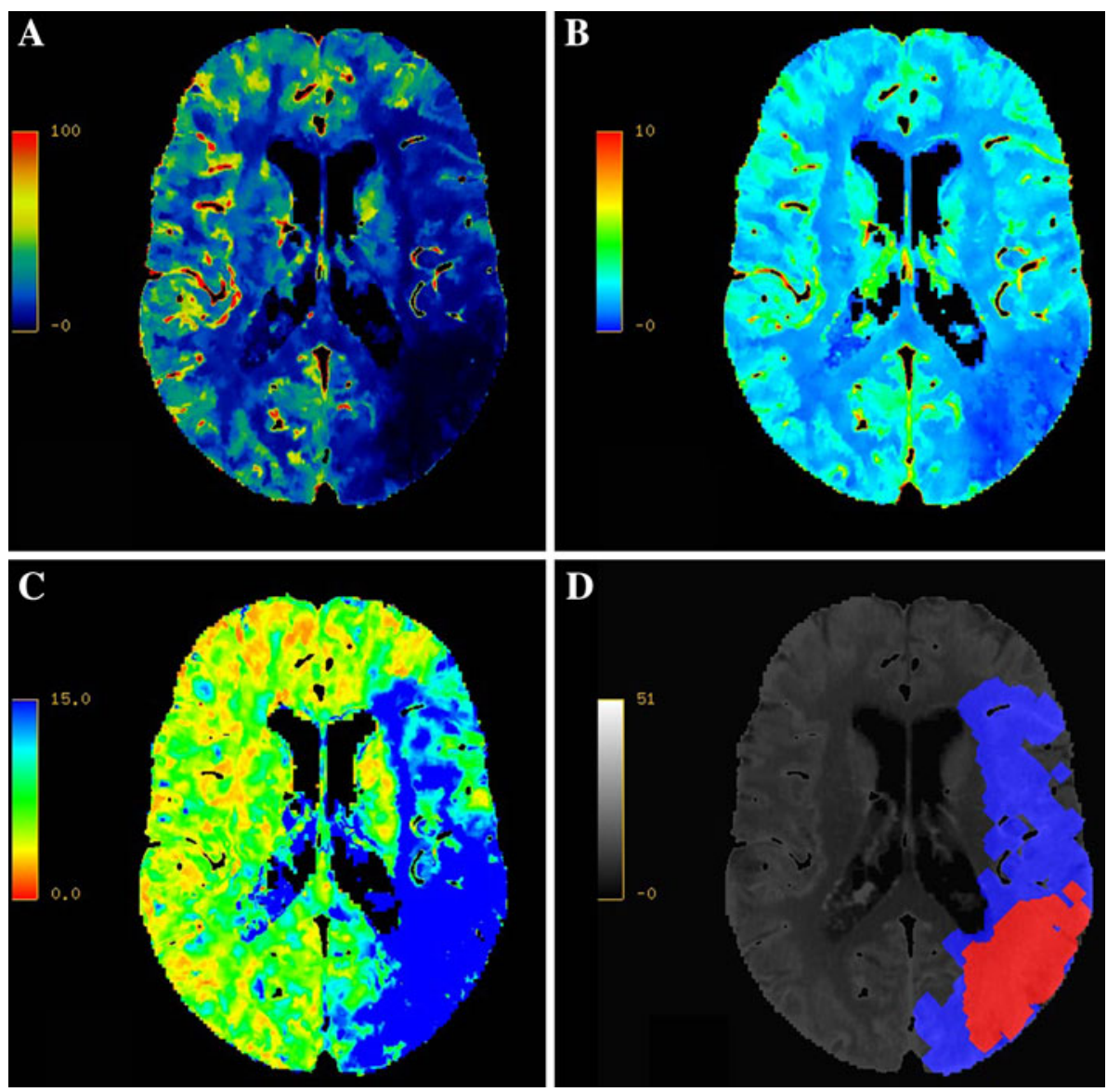
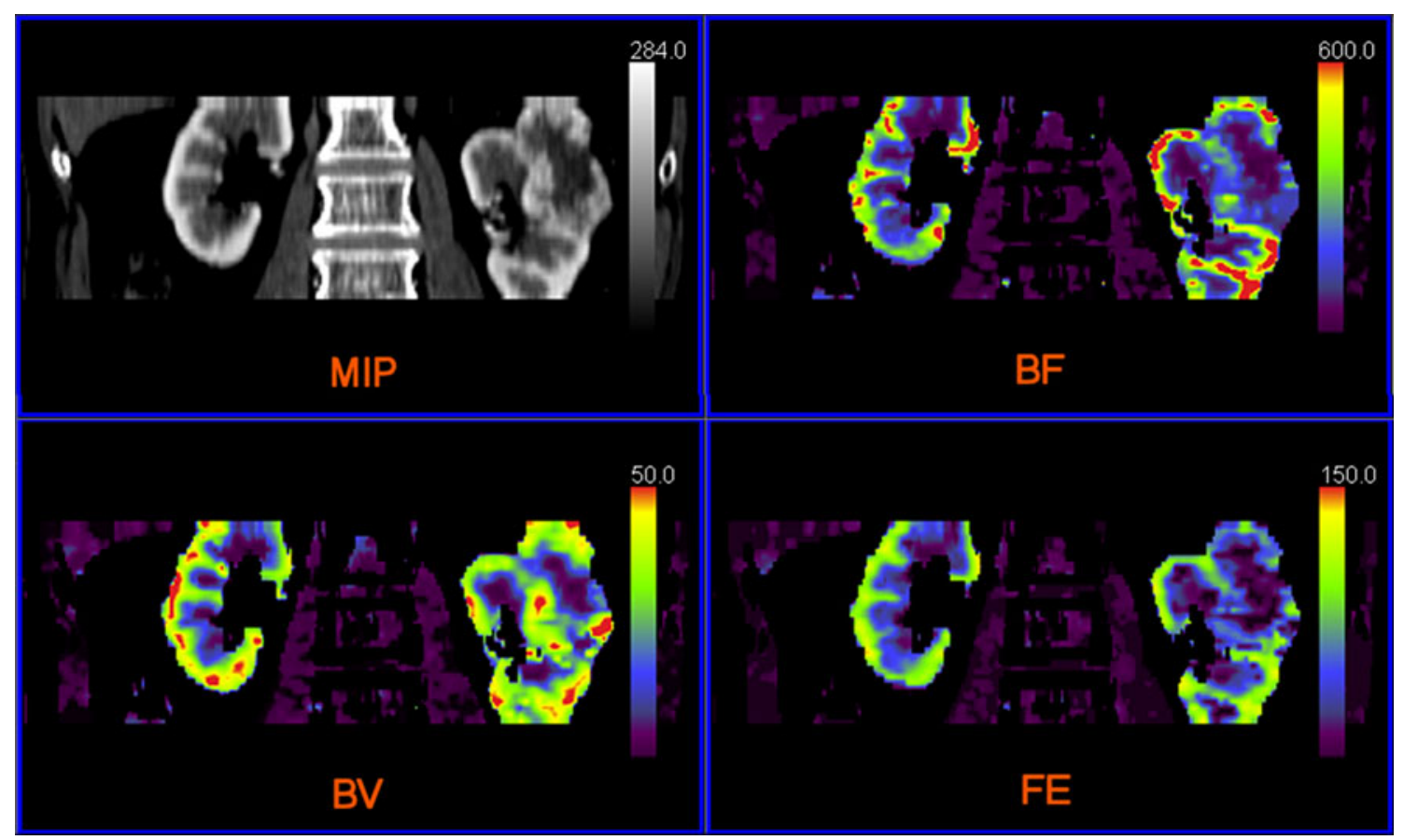

Fig. 5 Quantitative perfusion maps of a 76 year old man with renal cell carcinoma. Shown are coronal views of the temporal maximum projection, blood flow, blood volume and flow-extraction product.

Data were acquired in periodic spiral mode with $10 \mathrm{~cm}$ coverage. Parameter maps were calculated using a model based deconvolution technique [73]. Images courtesy of Siemens Medical Solutions 


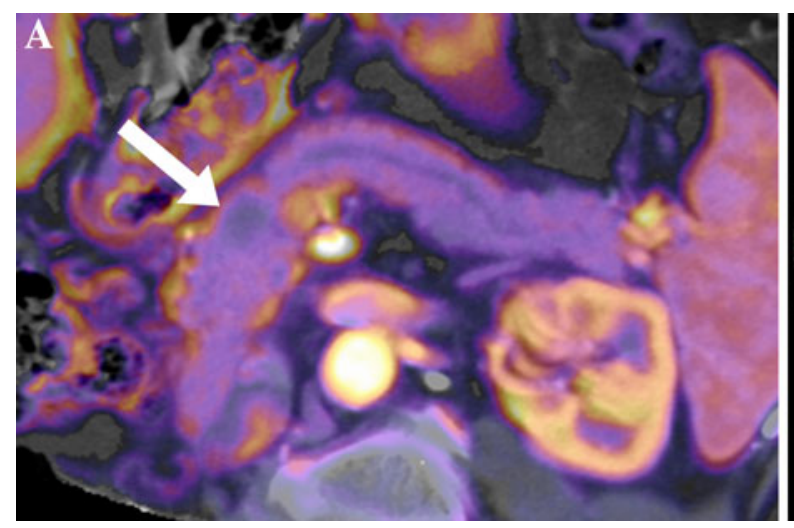

Fig. 6 Dynamic volume pancreatic perfusion showing a a well defined $1.4 \mathrm{~cm}$ mass in the pancreatic head (arrow) with upstream ductal dilatation is shown in this curved reconstruction. Low blood flow within the lesion $\mathbf{b}$ with values around $0.56 \mathrm{ml}^{-1}$. Whipple

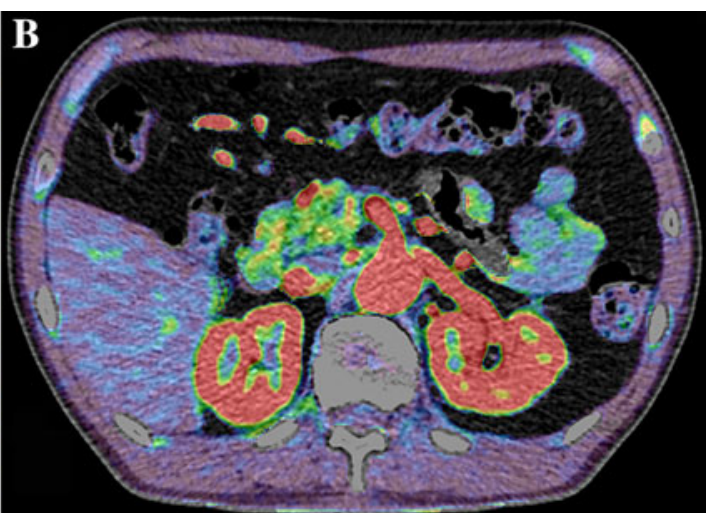

procedure was performed and pathology confirmed a pancreatic adenocarcinoma. Data were acquired in dynamic volume mode with $16 \mathrm{~cm}$ of coverage. Images courtesy of Patrik Rogalla
Fig. 7 Dynamically acquired demonstration of tracheomalacia at a full inspiratory phase showing normally expanded trachea and bronchial tree, $\mathbf{b}$ during forced expiration showing collapse of the posterior wall of the left bronchus and trachea, and $\mathbf{c}$ at full expiration with severe collapse of the trachea and total collapse of the bronchi. Data were acquired in dynamic volume mode with $16 \mathrm{~cm}$ of coverage. Images courtesy of Narinder Paul
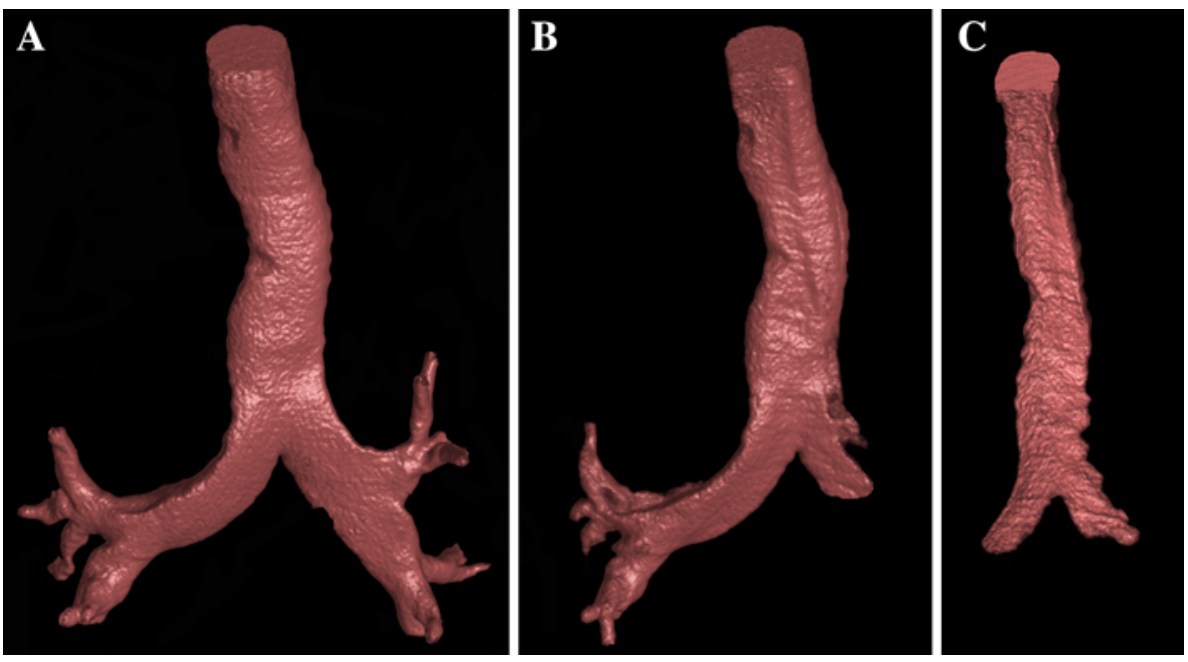

lower tube potential, typically 80 or $100 \mathrm{kV}$, can also lower the dose [94]. Furthermore, with the increase in iterative reconstruction and time-domain filtering techniques [95], the image quality of the reduced dose dynamic acquisitions can be greatly improved. Finally, as with all imaging, there are specific indications that benefit from a dynamic scan and others that do not, so care should be taken to use dynamic CT where it is most warranted.

There are even a few challenges inherent in the physiology and kinesiology of the body. For example, the field of dynamic MSK diagnostics is an emerging clinical field and the understanding about what "normal" motion is and what its boundaries are may not be completely known. Furthermore, many MSK pathologies are exacerbated by or only manifest under weight-bearing conditions. Simulating this can be difficult in most CT scanners, although one dedicated design has been proposed [96]. Similarly, for myocardial perfusion imaging, pharmacological stress agents must be employed to image the heart during hyperemia. Creating or simulating these situations can add to the challenge of dynamic imaging with $\mathrm{CT}$.

Another challenge with dynamic CT is the sheer volume of image data that is generated by these techniques. A 20 phase perfusion scan with 320 images per volume is over 6000 images. Therefore, the PACS systems, analysis packages, and infrastructure need to be prepared to handle larger volumes of data when dynamic scanning is involved. Furthermore, the software used to create these 4D images must reflect this new way of looking at form and function, and produce intuitive ways of managing and presenting this data to the reading clinician.

Ultimately, when used for the proper clinical indications, dynamic CT can be a useful and robust part of a diagnostic algorithm. Minimizing acquisition technique, reducing $\mathrm{kV}$ where appropriate, and employing iterative reconstruction and time-domain filtering provides a strong 
foundation for routine dynamic imaging. Furthermore, dynamic imaging plays a direct role in the effort to build patient-specific models of normal organ and body function [97]. CT will continue its expansion into the imaging of physiology and kinesiology. In this role, CT dynamics will be a cornerstone of functional imaging applications and will provide new insights into clinical diagnoses and improve patient care.

Disclosure R Mather is an employee of Toshiba Medical Research Institute USA, Inc.

\section{References}

Papers of particular interest, published recently, have been highlighted as:

- Of importance;

-. Of major importance

1. Mettler FA Jr, Thomadsen BR, Bhargavan M, et al. Medical radiation exposure in the U.S. in 2006: preliminary results. Health Phys. 2008;95:502-7.

2. Weintraub WS, Spertus JA, Kolm P, et al. Effect of PCI on quality of life in patients with stable coronary disease. N Engl J Med. 2008;359:677-87.

3. Tonino PA, De Bruyne B, Pijls NH, et al. FAME study investigators. Fractional flow reserve vs angiography for guiding percutaneous coronary intervention. N Engl J Med. 2009;360: 213-24.

4. • De Bruyne B, Pijls NHJ, Kalesan B, et al. Fractional flow reserve-guided PCI versus medical therapy in stable coronary disease. N Engl J Med. 2012;367:991-1001. Landmark trial showing that functional information (pressure drop across a lesion by FFR) can provide a better metric to drive therapy (PCI) than anatomic information (stenosis by conventional cath)

5. Ritman EL, Kinsey JH, Robb RA, et al. Physics and technical considerations in the design of the DSR: a high temporal resolution volume scanner. AJR. 1980;134:369-74.

6. Lipton MJ, Higgins CB, Farmer D, Boyd DP. Cardiac imaging with a high-speed Cine-CT Scanner: preliminary results. Radiology. 1984;152:579-82.

7. Iinuma TA, Tateno Y, Umegaki Y, Watanabe E. Proposed system for ultrafast computed tomography. J Comput Assist Tomogr. 1977;1:494-9.

8. Kachelriess M, Kalender WA. Electrocardiogram-correlated image reconstruction from subsecond spiral computed tomography scans of the heart. Med Phys. 1998;25:2417-31.

9. Taguchi K, Anno H. High temporal resolution for multislice helical computed tomography. Med Phys. 2000;27:861-72.

10. Achenbach S, Ulzheimer S, Baum U, et al. Noninvasive coronary angiography by retrospectively ECG-gated multislice spiral CT. Circulation. 2000;102:2823-8.

11. Ohnesorge B, Flohr T, Becker C, et al. Cardiac imaging by means of electrocardiographically gated multisection spiral CT: initial experience. Radiology. 2000;217:564-71.

12. Dinkel J, Welzel T, Bolte $\mathrm{H}$, et al. Four-dimensional multislice helical CT of the lung: qualitative comparison of retrospectively gated and static images in an ex vivo system. Radiother Oncol. 2007;85:215-22.
13. Christner JA, Stierstorfer K, Primak AN, et al. Evaluation of $\mathrm{z}$-axis resolution and image noise for nonconstant velocity spiral CT data reconstructed using a weighted 3D filtered backprojection (WFBP) reconstruction algorithm. Med Phys. 2010;37: 897-906.

14. Morhard D, Wirth CD, Fesl G, et al. Advantages of extended brain perfusion computed tomography: $9.6 \mathrm{~cm}$ coverage with time resolved computed tomography-angiography in comparison to standard stroke-computed tomography. Invest Radiol. 2010;45: 363-9.

15. Rybicki FJ, Otero HJ, Steigner ML, et al. Initial evaluation of coronary images from 320-detector row computed tomography. Int J Cardiovasc Imaging. 2008;24:535-46.

16. Bardo DM, Asamato J, Mackay CS, Minette M. Low-dose coronary artery computed tomography angiogram of an infant with tetralogy of fallot using a 256-slice multidetector computed tomography scanner. Pediatr Cardiol. 2009;30:824-6.

17. San Millán Ruíz D, Murphy K, Gailloud P. 320-Multidetector row whole-head dynamic subtracted CT angiography and wholebrain CT perfusion before and after carotid artery stenting: technical note. Eur J Radiol. 2009;74:413-9.

18. Werner FW, Short WH, Green JK. Changes in patterns of scaphoid and lunate motion during functional arcs of wrist motion induced by ligament division. J Hand Surg Am. 2005;30:1156-60.

19. Braunstein EM, Louis DS, Greene TL, et al. Fluoroscopic and arthrographic evaluation of carpal instability. AJR. 1985;144: 1259-62.

20. Hentz VR, Marich KW, Dev P. Preliminary study of the upper limb with the use of ultrasound transmission imaging. J Hand Surg Am. 1984;9:188-93.

21. Bond JR, Berquist TH. Radiologic evaluation of hand and wrist motion. Hand Clin. 1991;7:113-23.

22. Stanford W, Phelan J, Kathol MH, et al. Patellofemoral joint motion: evaluation by ultrafast computed tomography. Skeletal Radiol. 1988;17:487-92.

23. Shapeero LG, Dye SF, Lipton MJ, et al. Functional dynamics of the knee joint by ultrafast, cine-CT. Invest Radiol. 1988;23: 118-23.

24. Tay SC, Primak AN, Fletcher JG, et al. Understanding the relationship of image quality and motion velocity in gated-CT imaging: preliminary work for 4D musculoskeletal imaging. J Comput Assist Tomogr. 2008;32:634-9.

25. Tay SC, Primak AN, Fletcher JG, et al. Four-dimensional computed tomographic imaging in the wrist: proof of feasibility in a cadaveric model. Skeletal Radiol. 2007;36:1163-9.

26. Leng S, Zhao K, Qu M, et al. Dynamic CT technique for assessment of wrist joint instabilities. Med Phys. 2011;38(Suppl 1):S50.

27. - Kalia V, Obray RW, Filice R, et al. Functional joint imaging using 256-MDCT: technical feasibility. AJR Am J Roentgenol. 2009;192:W295-9. The first report on area detector CT imaging of joints in motion. The first report on area detector CT imaging of joints in motion

28. Halpenny D, Courtney K, Torreggiani WC. Dynamic fourdimensional 320 section CT and carpal bone injury - a description of a novel technique to diagnose scapholunate instability. Clin Radiol. 2012;67:185-7.

29. Wassilew GI, Janz V, Heller MO, et al. Real time visualization of femoroacetabular impingement and subluxation using 320-slice computed tomography. J Orthop Res. 2012;. doi:10.1002/jor.22224.

30. Klingebiel R, Siebert E, Diekmann S, et al. 4-D imaging in cerebrovascular disorders by using 320-slice CT: feasibility and preliminary clinical experience. Acad Radiol. 2009;16:123-9.

31. Stavropoulos AW, Clark TW, Carpenter JP, et al. Use of CT angiography to classify endoleaks after endovascular repair of abdominal aortic aneurysms. J Vasc Interv Radiol. 2005;16: $663-7$. 
32. - Bent CL, Jaskolka JD, Lindsay TF, Tan K. The use of dynamic volumetric CT angiography (DV-CTA) for the characterization of endoleaks following fenestrated endovascular aortic aneurysm repair (f-EVAR). J Vasc Surg. 2010;51:203-6. One of the first reports on the use of dynamic volume CT angiography. Shows that dynamic assessment of endoleaks can improve characterization.

33. Brouwer PA, Bosman T, van Walderveen MA, et al. Dynamic 320-section CT angiography in cranial arteriovenous shunting lesions. AJNR. 2010;31:767-70.

34. Willems PW, Taeshineetanakul P, Schenk B, et al. The use of 4D-CTA in the diagnostic work-up of brain arteriovenous malformations. Neuroradiology. 2012;54:123-31.

35. Willems PW, Brouwer PA, Barfett JJ, et al. Detection and classification of cranial dural arteriovenous fistulas using 4D-CT angiography: initial experience. AJNR Am J Neuroradiol. 2011; 32:49-53.

36. Krings T, Willems P, Barfett J, et al. Pulsatility of an intracavernous aneurysm demonstrated by dynamic 320-detector row CTA at high temporal resolution. Cent Eur Neurosurg. 2009;70: 214-8.

37. Meinel FG, Nikolaou K, Weidenhagen R, et al. Time-resolved CT angiography in aortic dissection. Eur J Radiol. 2012;81: 3254-61.

38. - Sommer WH, Clevert DA, Bamberg F, et al. Time-resolved computed tomography imaging of the aorta: a feasibility study. J Thorac Imaging. 2010;25:161-7. Demonstrated feasibility of helical shuttle to produce time-resolved CTA over a wide area.

39. Sommer WH, Becker CR, Haack M, et al. Time-resolved CT angiography for the detection and classification of endoleaks. Radiology. 2012;263:917-26.

40. Barfett JJ, Fierstra J, Mikulis DJ, Krings T. Blood velocity calculated from volumetric dynamic computed tomography angiography. Invest Radiol. 2010;45:778-81.

41. Shpilfoygel SD, Close RA, Valentino DJ, et al. X-ray videodensitometric methods for blood flow and velocity measurement: a critical review of literature. Med Phys. 2000;27:2008-23.

42. Axel L. Cerebral blood flow determination by rapid-sequence computed tomography: theoretical analysis. Radiology. 1980; 137:679-86.

43. Wolfkiel CJ, Ferguson JL, Chomka EV, et al. Measurement of myocardial blood flow by ultrafast computed tomography. Circulation. 1987;76:1262-73.

44. Rumberger JA, Feiring AJ, Lipton MJ, et al. Use of ultrafast computed tomography to quantitate regional myocardial perfusion: a preliminary report. JACC. 1987;9:59-69.

45. Parsons MW, Pepper EM, Chan V, et al. Perfusion computed tomography: prediction of final infarct extent and stroke outcome. Ann Neurol. 2005;58:672-9.

46. Kohrmann M, Juttler E, Fiebach JB, et al. MRI versus CT-based thrombolysis treatment within and beyond the $3 \mathrm{~h}$ time window after stroke onset: a cohort study. Lancet Neurol. 2006;5:661-7.

47. Schramm P, Schellinger PD, Klotz E, et al. Comparison of perfusion computed tomography and computed tomography angiography source images with perfusion-weighted imaging and diffusion weighted imaging in patients with acute stroke of less than 6 hours' duration. Stroke. 2004;35:1652-8.

48. Wintermark M, Reichhart M, Cuisenaire O, et al. Comparison of admission perfusion computed tomography and qualitative diffusion- and perfusion-weighted magnetic resonance imaging in acute stroke patients. Stroke. 2002;33:2025-31.

49. Konstas AA, Wintermark M, Lev MH. CT perfusion imaging in acute stroke. Neuroimaging Clin N Am. 2011;21:215-38. ix.

50. Salomon EJ, Barfett J, Willems PW, et al. Dynamic CT angiography and CT perfusion employing a 320-detector row CT: protocol and current clinical applications. Klin Neuroradiol. 2009;19(3):187-96.
51. Dabaneh H, Guerrero W, Wilson K, et al. Observation of Mean Transit Time (Mtt) perfusion maps on a 320-Detector Row CT Scanner and its potential application in Acute Ischemic Stroke. J Neurol Neurophysiol. 2011;2:115.

52. Dorn F, Muenzel D, Meier R, et al. Brain perfusion CT for acute stroke using a 256-slice CT: improvement of diagnostic information by large volume coverage. Eur Radiol. 2011;21:1803-10.

53. Roberts HC, Roberts TP, Smith WS, et al. Multisection dynamic CT perfusion for acute cerebral ischemia: the "toggling-table" technique. AJNR. 2001;22:1077-80.

54. Saake M, Goelitz P, Struffert T, et al. Comparison of Conventional CTA and Volume Perfusion CTA in Evaluation of Cerebral Arterial Vasculature in Acute Stroke. AJNR Am J Neuroradiol. 2012;33(11):2068-73.

55. Zhong L, Wang WJ, Xu JR. Clinical application of hepatic CT perfusion. World J Gastroenterol. 2009;15:907-11.

56. Kanda T, Yoshikawa T, Ohno Y, et al. Perfusion measurement of the whole upper abdomen of patients with and without liver diseases: initial experience with 320-detector row CT. Eur J Radiol. 2012;81:2470-5.

57. Ng CS, Chandler AG, Wei W, et al. Reproducibility of CT perfusion parameters in liver tumors and normal liver. Radiology. 2011;260:762-70.

58. Beganovic A, Sefic-Pasic I, Skopljak-Beganovic A, et al. Doses to skin during dynamic perfusion computed tomography of the liver. Radiat Prot Dosimetry. 2013;153:106-11.

59. Watanabe S, Katada Y, Gohkyu M, et al. Liver perfusion CT during hepatic arteriography for the hepatocellular carcinoma: Dose reduction and quantitative evaluation for normal- and ultralow-dose protocol. Eur J Radiol. 2012;81(12):3993-7.

60. Katada Y, Shukuya T, Kawashima M, et al.: A comparative study between arterial spin labeling and CT perfusion methods on hepatic portal venous flow. Jpn J Radiol. 2012 [Epub ahead of print].

61. Kanda T, Yoshikawa T, Ohno Y, et al. CT hepatic perfusion measurement: comparison of three analytic methods. Eur $\mathrm{J}$ Radiol. 2012;81:2075-9.

62. Miles KA, Hayball MP, Dixon AK. Measurement of human pancreatic perfusion using dynamic computed tomography with perfusion imaging. Br J Radiol. 1995;68:471-5.

63. Kandel S, Kloeters C, Meyer H, et al. Whole-organ perfusion of the pancreas using dynamic volume $\mathrm{CT}$ in patients with primary pancreas carcinoma: acquisition technique, post-processing and initial results. Eur Radiol. 2009;19:2641-6.

64. Delrue L, Blanckaert P, Mertens D, et al. Assessment of tumor vascularization in pancreatic adenocarcinoma using 128-slice perfusion computed tomography imaging. J Comput Assist Tomogr. 2011;35:434-8.

65. Klauss M, Stiller W, Fritz F, et al. Computed tomography perfusion analysis of pancreatic carcinoma. J Comput Assist Tomogr. 2012;36:237-42.

66. Zamboni GA, Bernardin L. Pozzi Mucelli R: dynamic MDCT of the pancreas: is time-density curve morphology useful for the differential diagnosis of solid lesions? A preliminary report. Eur J Radiol. 2012;81:e381-5.

67. Motosugi U, Ichikawa $\mathrm{T}$, Sou $\mathrm{H}$, et al. Multi-organ perfusion $\mathrm{CT}$ in the abdomen using a 320-detector row CT scanner: preliminary results of perfusion changes in the liver, spleen, and pancreas of cirrhotic patients. Eur J Radiol. 2012;81:2533-7.

68. - Ohno Y, Koyama H, Matsumoto K, et al. Differentiation of malignant and benign pulmonary nodules with quantitative firstpass 320-detector row perfusion CT versus FDG PET/CT. Radiology. 2011;258:599-609. The first study of dynamic volume perfusion in the lung. Showed ability of perfusion to identify malignant nodules.

69. Sauter AW, Merkle A, Schulze M, et al. Intraobserver and interobserver agreement of volume perfusion CT (VPCT) 
measurements in patients with lung lesions. Eur $\mathrm{J}$ Radiol. 2012;81:2853-9.

70. Iwano S, Koike W, Matsuo K, et al. Correlation between dynamic CT findings and pathological prognostic factors of small lung adenocarcinoma. Cancer Imaging. 2012;12:187-93.

71. Ng CS, Chandler AG, Wei W, et al. Reproducibility of perfusion parameters obtained from perfusion CT in lung tumors. AJR Am J Roentgenol. 2011;197:113-21.

72. Lemoine S, Papillard M, Belloi A, et al. Renal perfusion: noninvasive measurement with multidetector CT versus fluorescent microspheres in a pig model. Radiology. 2011;260:414-20.

73. Reiner CS, Goetti R, Eberli D, et al. CT perfusion of renal cell carcinoma: impact of volume coverage on quantitative analysis. Invest Radiol. 2012;47:33-40.

74. Ng CS, Wang X, Faria SC, Lin E, et al. Perfusion CT in patients with metastatic renal cell carcinoma treated with interferon. AJR Am J Roentgenol. 2010;194:166-71.

75. Tsushima Y, Unno Y, Koizumi J, Kusano S. Measurement of human hepatic and splenic perfusion using dynamic computed tomography: a preliminary report. Comput Methods Programs Biomed. 1998;57:143-6.

76. Sauter AW, Feldmann S, Spira D, et al. Assessment of splenic perfusion in patients with malignant hematologic diseases and spleen involvement, liver cirrhosis and controls using volume perfusion CT (VPCT): a pilot study. Acad Radiol. 2012;19: 579-87.

77. Dighe S, Castellano E, Blake H, et al. Perfusion CT to assess angiogenesis in colon cancer: technical limitations and practical challenges. Br J Radiol. 2012;85:e814-25.

78. Goh V, Rodriguez-Justo M, Engledow A, et al. Assessment of the metabolic flow phenotype of primary colorectal cancer: correlations with microvessel density are influenced by the histological scoring method. Eur Radiol. 2012;22:1687-92.

79. Kim JW, Jeong YY, Chang NK, et al. Perfusion CT in colorectal cancer: comparison of perfusion parameters with tumor grade and microvessel density. Korean J Radiol. 2012;13(Suppl 1):S89-97.

80. Wang Y, Qin L, Shi X, et al. Adenosine-stress dynamic myocardial perfusion imaging with second-generation dual-source CT: comparison with conventional catheter coronary angiography and SPECT nuclear myocardial perfusion imaging. AJR Am J Roentgenol. 2012;198:521-9.

81. Bastarrika G, Ramos-Duran L, Rosenblum MA, et al. Adenosinestress dynamic myocardial CT perfusion imaging: initial clinical experience. Invest Radiol. 2010;45:306-13.

82. George RT, Arbab-Zadeh A, Cerci RJ, et al. Diagnostic performance of combined noninvasive coronary angiography and myocardial perfusion imaging using 320-MDCT: the ct angiography and perfusion methods of the CORE320 multicenter multinational diagnostic study. AJR Am J Roentgenol. 2011; 197:829-37.
83. Ko BS, Cameron JD, Meredith IT, et al. Computed tomography stress myocardial perfusion imaging in patients considered for revascularization: a comparison with fractional flow reserve. Eur Heart J. 2012;33:67-77.

84. Blankstein R, Shturman LD, Rogers IS, et al. Adenosine-induced stress myocardial perfusion imaging using dual-source cardiac computed tomography. J Am Coll Cardiol. 2009;54:1072-84.

85. van Beek EJ, Hoffman EA. Functional imaging: CT and MRI. Clin Chest Med. 2008;29:195-216.

86. Lee KS, Sun MR, Ernst A, et al. Comparison of dynamic expiratory $\mathrm{CT}$ with bronchoscopy for diagnosing airway Malacia: a pilot evaluation. Chest. 2007;131:758-64.

87. Wagnetz U, Roberts HC, Chung T, et al. Dynamic airway evaluation with volume CT: initial experience. Can Assoc Radiol J. 2010;61:90-7.

88. Low K, Lau KK, Holmes P, et al. Abnormal vocal cord function in difficult-to-treat asthma. Am J Respir Crit Care Med. 2011; 184:50-6.

89. Helm E, Talakoub O, Grasso F, et al. Use of dynamic CT in acute respiratory distress syndrome (ARDS) with comparison of positive and negative pressure ventilation. Eur Radiol. 2009;19:50-7.

90. Ding K, Cao K, Fuld MK, et al. Comparison of image registration based measures of regional lung ventilation from dynamic spiral CT with Xe-CT. Med Phys. 2012;39:5084-98.

91. Coolens C, Bracken J, Driscoll B, et al. Dynamic volume vs respiratory correlated 4DCT for motion assessment in radiation therapy simulation. Med Phys. 2012;39:2669-81.

92. Underberg RW, Lagerwaard FJ, Cuijpers JP, et al. Four-dimensional CT scans for treatment planning in stereotactic radiotherapy for stage I lung cancer. Int J Radiat Oncol Biol Phys. 2004; 60:1283-90.

93. Greenberg SB. Dynamic pulmonary CT of children. AJR Am J Roentgenol. 2012;199:435-40.

94. Nakayama Y, Awai K, Funama Y, et al. Abdominal CT with low tube voltage: preliminary observations about radiation dose, contrast enhancement, image quality, and noise. Radiology. 2005; 237:945-51.

95. Mendrik AM, Vonken EJ, van Ginneken B, et al. TIPS bilateral noise reduction in $4 \mathrm{D} C \mathrm{C}$ perfusion scans produces high-quality cerebral blood flow maps. Phys Med Biol. 2011;56:3857-72.

96. Zbijewski W, De Jean P, Prakash P, et al. A dedicated cone-beam CT system for musculoskeletal extremities imaging: design, optimization, and initial performance characterization. Med Phys. 2011;38:4700-13.

97. Mihalef V, Ionasec RI, Sharma P, et al. Patient-specific modelling of whole heart anatomy, dynamics and hemodynamics from fourdimensional cardiac CT images. Interface Focus. 2011;1:286-96. 